\title{
Holographic Complex Conformal Field Theories
}

\author{
Antón F. Faedo $\odot,{ }^{1}$ Carlos Hoyos $\odot{ }^{2,3}$ David Mateos $\odot,{ }^{1,4}$ and Javier G. Subils $\oplus^{1}$ \\ ${ }^{1}$ Departament de Física Quàntica i Astrofísica \& Institut de Ciències del Cosmos (ICC), Universitat de Barcelona, \\ Martí i Franquès 1, 08028 Barcelona, Spain \\ ${ }^{2}$ Deptartment of Physics, Universidad de Oviedo, Federico García Lorca 18, 33007 Oviedo, Spain \\ ${ }^{3}$ Instituto Universitario de Ciencias y Tecnologías Espaciales de Asturias (ICTEA), Universidad de Oviedo, \\ Calle de la Independencia, 13, 33004 Oviedo, Spain \\ ${ }^{4}$ Institució Catalana de Recerca i Estudis Avançats (ICREA), Lluís Companys 23, 08010 Barcelona, Spain
}

(Received 8 November 2019; revised manuscript received 3 February 2020; accepted 24 March 2020; published 20 April 2020)

\begin{abstract}
The loss of criticality in the form of weak first-order transitions or the end of the conformal window in gauge theories can be described as the merging of two fixed points that move to complex values of the couplings. When the complex fixed points are close to the real axis, the system typically exhibits walking behavior with Miransky (or Berezinsky-Kosterlitz-Thouless) scaling. We present a novel realization of these phenomena at strong coupling by means of the gauge/gravity duality, and give evidence for the conjectured existence of complex conformal field theories at the fixed points.
\end{abstract}

DOI: 10.1103/PhysRevLett.124.161601

Introduction.-Fixed-point annihilation (FPA) is an interesting phenomenon in which two fixed points (FPs) of the renormalization group (RG) flow merge and disappear as some parameter is varied. In the context of phase transitions and critical phenomena it is associated to a change from continuous to weak first-order transitions. Examples include the superconducting transition in the Abelian Higgs model $[1,2]$, the related Néel-valence bond-solid transition in antiferromagnets [3-6], the ferromagnetic transition in the Potts model [7-9], metal-Mott insulator transitions [10] and six-dimensional $O(N)$ models $[11,12]$. FPA has also been associated to the boundaries of the conformal window in gauge theories with flavors, both in $(2+1)$-dimensional quantum electrodynamics [13-16] and in non-Abelian gauge theories in $3+1$ dimensions [17-21].

More generally, FPA has been proposed as a natural mechanism to produce "walking behavior" in gauge theories [22]. The idea is that, just after the merging, the critical points leave a footprint in the form of approximate scale invariance over a large range of scales. Typically the range of the walking region increases exponentially as parameters are tuned to the merging point, following Miransky (or Berezinsky-Kosterlitz-Thouless) scaling [23-25]. This behavior can be explained by continuing the theory to complex values of the couplings, so that the annihilation is understood as a migration of the FPs to the complex plane after the merger [19]. Their effect on the RG flow is

Published by the American Physical Society under the terms of the Creative Commons Attribution 4.0 International license. Further distribution of this work must maintain attribution to the author(s) and the published article's title, journal citation, and DOI. Funded by SCOAP ${ }^{3}$. noticeable as long as they remain close to the real axis. It has been recently conjectured [22] that a nonunitary, complex conformal field theory (CCFT) exists at each of the two complex fixed points (CFPs), so that the properties of the theory in the walking region can be derived from perturbations of the CCFTs. Each CCFT has a complex spectrum of operators that is the conjugate of its companion's, implying that CFPs should always come in pairs.

Although FPA and CFPs are expected to exist generically, their study has been mostly limited to weakly coupled theories (see, e.g., [26-29] for recent examples), as their identification requires computing the beta functions for the different couplings in the theory, a task that often can only be done via perturbation theory. In this paper we will construct a simple holographic model that realizes FPA and CFPs, thus showing that these phenomena can also occur at strong coupling. In addition, our analysis provides nonperturbative evidence that CFPs have the conjectured properties of CCFTs regarding the spectrum of local operators.

Fixed-point annihilation and complex CFTs.-Consider a system with a dimensionless coupling $g$ whose $\beta$ function depends on an external parameter $\alpha$ in such a way that, for $\alpha \simeq \alpha_{*}$,

$$
\beta(g) \simeq\left(\alpha-\alpha_{*}\right)-\left(g-g_{*}\right)^{2} .
$$

We will see an explicit example in the next section. If $\alpha>\alpha_{*}$, discarding higher-order terms, the $\beta$ function vanishes at two values

$$
g_{ \pm}=g_{*} \pm \sqrt{\alpha-\alpha_{*}} .
$$

To make sure that the theory is well defined in the far ultraviolet (UV) we may imagine that $\beta$ has another zero at 
some $g_{\infty}<g_{-}$. Decreasing the control parameter $\alpha$ the FPs $g_{ \pm}$approach each other until they merge at $\alpha=\alpha_{*}$. If we decrease $\alpha$ further, $\beta(g)$ loses these (real) zeroes and the theory ceases to have a (real) conformal phase in the infrared (IR). However, for $\left|\alpha-\alpha_{*}\right|$ sufficiently small, $\beta(g)$ has CFPs close to the real axis at $g_{ \pm}=g_{*} \pm i \sqrt{\alpha_{*}-\alpha}$. In this regime the theory exhibits approximate scale invariance between UV and IR scales $\mu_{\mathrm{UV}}$ and $\mu_{\mathrm{IR}}$ defined by the values of the coupling $g_{\mathrm{UV}} \lesssim g_{*} \lesssim g_{\mathrm{IR}}$. The ratio between these two scales becomes exponentially large as $\alpha$ approaches $\alpha_{*}$ and shows the characteristic Miransky scaling

$$
\log \frac{\mu_{\mathrm{UV}}}{\mu_{\mathrm{IR}}}=\int_{g_{\mathrm{IR}}}^{g_{\mathrm{UV}}} \frac{d g}{\beta(g)} \simeq \frac{\pi}{\sqrt{\alpha_{*}-\alpha}},
$$

where we assumed that $\left|g_{\mathrm{IR}, \mathrm{UV}}-g_{*}\right| \gg \sqrt{\alpha_{*}-\alpha}$. Thus if $\left|\alpha-\alpha_{*}\right|$ is small then the RG flow is slow in a large energy range, hence the term "walking" flow.

The scenario conjectured in $[9,22]$ is that CFPs correspond to pairs of nonunitary CCFTs that control the walking flow, which passes precisely in between them. Each CCFT should have operators of complex dimensions in the spectrum that are not matched by other operators with complex conjugate dimension in the same theory. Instead, the missing operators of complex conjugate dimensions in one CCFT should be part of the spectrum of the companion CCFT. Thus in this sense the two CCFTs are complex conjugates of one another.

An important case is the operator associated to the coupling $g$ itself, whose complex dimensions at each CFP,

$$
\Delta_{ \pm}=d+\beta^{\prime}\left(g_{ \pm}\right) \simeq d \mp 2 i \sqrt{\alpha_{*}-\alpha},
$$

with $d$ as the spacetime dimension, are indeed complex conjugates of one another. Moreover, this operator is close to marginality when $\alpha \lesssim \alpha_{*}$, with the leading deviation being imaginary and small. Using this, the hierarchy (3) can be rewritten as

$$
\log \frac{\mu_{\mathrm{UV}}}{\mu_{\mathrm{IR}}} \simeq \frac{2 \pi}{|\operatorname{Im} \Delta|}
$$

Holographic realization.-Previous holographic realizations of walking behavior with Miransky-like scaling in gravity duals [19,30-33] are based on flows where the mass of a scalar field on the gravity side violates the Breitenlohner-Freedman (BF) bound [34]. In these cases there is a real FP that becomes dynamically unstable but no CFPs have been identified. We will present a different construction in which both FPA and the resulting CFPs are explicitly realized.

Couplings of the gauge theory are holographically dual to fields on the gravity side. For simplicity we focus on a single coupling dual to a scalar field. The action on the gravity side is thus

$$
S=\frac{1}{2 \kappa^{2}} \int d^{d+1} x \sqrt{-g}\left(R-\frac{1}{2}(\partial \phi)^{2}-V(\phi)\right) .
$$

For each critical point $\phi_{c}$ of the potential with $V\left(\phi_{c}\right)<0$ there is an anti-de Sitter (AdS) solution with its corresponding $d$-dimensional CFT dual. We choose to write $V$ in terms of a (fake) superpotential $W$ through the usual relation

$$
V=(d-1)\left[2(d-1)\left(\frac{d W}{d \phi}\right)^{2}-d W^{2}\right] .
$$

The only reason for this is to simplify the presentation. In particular, this choice implies nothing regarding the possible presence of supersymmetry in the system. Critical points of $W$ are also critical points of $V$ (but not viceversa). Since we wish to model three FPs we take a superpotential with derivative

$$
\frac{d W}{d \phi}=\frac{W_{0}}{L} \phi\left(\phi-\phi_{0}\right)\left(\phi-\bar{\phi}_{0}\right) .
$$

The resulting potential is shown in Fig. 1. The UV FP dual to the AdS solution at $\phi=0$ is the analog of the FP at $g=g_{\infty}$ in the previous section. The constants $\phi_{0}$ and $\bar{\phi}_{0}$ are parameters of the model analogous to $\alpha$. If both $\phi_{0}$ and $\bar{\phi}_{0}$ are real then there are two additional real FPs at $\phi=\phi_{0}$ and $\phi=\bar{\phi}_{0}$, in analogy with $g=g_{ \pm}$in the previous section. When $\phi_{0}=\bar{\phi}_{0}$ these FPs merge into a single one. If $\phi_{0}$ and $\bar{\phi}_{0}$ become complex then they must be conjugate to one another since $W$ must be real for real $\phi$. In this case the potential looses two real critical points, giving a holographic realization of FPA [37].

At the UV FP the AdS radius is fixed by the integration constant $W(0)=1 / L$, while the dimension $\Delta_{\mathrm{UV}}$ of the operator $\mathcal{O}$ dual to $\phi$ is determined by $W_{0}=$ $\delta_{\mathrm{UV}} /\left[2(d-1) \phi_{0} \bar{\phi}_{0}\right]>0$. There are two possible choices depending on whether the flow is triggered by a source for

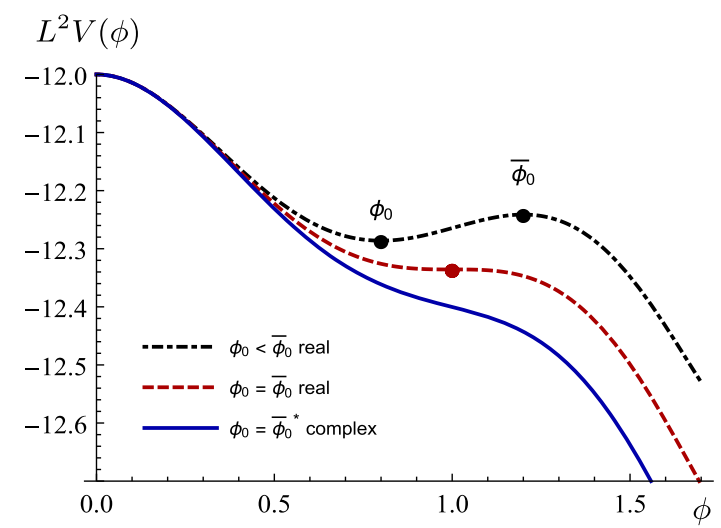

FIG. 1. Potential of our model for the cases $\left\{\phi_{0}=0.8\right.$, $\left.\bar{\phi}_{0}=1.2\right\}$ (top curve), $\left\{\phi_{0}=\bar{\phi}_{0}=1\right\}$ (middle curve), and $\left\{\phi_{0}=\bar{\phi}_{0}^{*}=1+0.2 i\right\}$ (bottom curve). 
$\mathcal{O}$, in which case $\delta_{\mathrm{UV}}=d-\Delta_{\mathrm{UV}}$, or by a nonzero expectation value for $\mathcal{O}$, in which case $\delta_{\mathrm{UV}}=\Delta_{\mathrm{UV}}$.

Expanding the superpotential around $\phi=\phi_{0}$ we get

$W(\phi)=\frac{1}{L_{0}}+\frac{\delta_{0}}{4(d-1) L_{0}}\left(\phi-\phi_{0}\right)^{2}+O\left(\phi-\phi_{0}\right)^{3}$,

where

$$
L_{0}=L\left[1+\frac{W_{0}}{6}\left(\phi_{0}^{3} \bar{\phi}_{0}-\frac{\phi_{0}^{4}}{2}\right)\right]^{-1}
$$

is the AdS radius at $\phi=\phi_{0}$ and

$\delta_{0}=2(d-1) W_{0} \frac{L_{0}}{L} \phi_{0}\left(\phi_{0}-\bar{\phi}_{0}\right)=\delta_{\mathrm{UV}} \frac{L_{0}}{L} \frac{\left(\phi_{0}-\bar{\phi}_{0}\right)}{\bar{\phi}_{0}}$.

The expansion around $\bar{\phi}_{0}$ gives analogous results with the replacements $\left\{\phi_{0}, \bar{\phi}_{0}, L_{0}, \delta_{0}\right\} \rightarrow\left\{\bar{\phi}_{0}, \phi_{0}, \bar{L}_{0}, \bar{\delta}_{0}\right\}$. Assuming $0<\phi_{0} \lesssim \bar{\phi}_{0}$ we have that

$$
\delta_{0}<0, \quad 0<\bar{\delta}_{0}<\frac{d}{2}-1 .
$$

In this case $\bar{\phi}_{0}$ corresponds to an UV FP deformed by a relevant scalar operator of dimension $\bar{\Delta}_{0}=d-\bar{\delta}_{0}$, whereas $\phi_{0}$ corresponds to an IR FP deformed by an irrelevant scalar operator of dimension $\Delta_{0}=d-\delta_{0}$. When $\phi_{0}=\bar{\phi}_{0}$ the two points merge and the dual operator becomes marginal.

Complex FPs and RG flows: In the so-called domain wall coordinates in which the metric takes the form

$$
d s_{d+1}^{2}=g_{M N} d x^{M} d x^{N}=e^{2 A(\rho)} d x_{1, d-1}^{2}+d \rho^{2}
$$

the solution is determined by the equations

$$
\frac{d A}{d \rho}=W, \quad \frac{d \phi}{d \rho}=-2(d-1) \frac{d W}{d \phi} .
$$

In these coordinates the metric is foliated by copies of $d$-dimensional Minkowski space with scale factor $e^{A(\rho)}$, which is therefore interpreted as dual to the RG scale in the gauge theory. Similarly, the scalar field $\phi=\phi(\rho)$ is dual to a running coupling constant in a particular scheme, whose $\beta$ function is therefore (see, e.g., [38])

$$
\beta(\phi)=\frac{d \phi}{d A}=-2(d-1) \frac{d \log W}{d \phi} .
$$

Close to any of the three real FPs $\phi_{c}=\left\{0, \phi_{0}, \bar{\phi}_{0}\right\}$ one finds the expected behavior

$$
\phi \simeq g_{c} e^{-\left(d-\Delta_{c}\right) \rho / L_{c}}, \quad A(\rho) \simeq \frac{\rho}{L_{c}} \sim \log \frac{\mu}{\Lambda_{c}},
$$

and

$$
\beta(\phi) \simeq-\left(d-\Delta_{c}\right)\left(\phi-\phi_{c}\right)+O\left[\left(\phi-\phi_{c}\right)^{2}\right],
$$

where $\mu$ is the RG scale, $\Lambda_{c}$ is the scale that triggers the flow away or into the FP, and $g_{c}$ is the corresponding (dimensionless) coupling at the FP. UV and IR FPs are approached for $\rho \rightarrow \infty$ and $\rho \rightarrow-\infty$, respectively. The $\beta$ functions for our model are shown in Fig. 2, where we see that they exhibit the behavior discussed in the previous section.

Since the scalar field is dual to the coupling constant in the gauge theory, we propose that the holographic dual of the extension of this coupling to complex values consists of extending the scalar field on the gravity side to complex values as well. Since the scalar field couples to the metric, we also extend the metric components to complex values. We assume that, in this extension, the action (6) is a holomorphic function of $g_{M N}$ and $\phi$. In other words, we do not introduce any explicit dependence on the complex conjugates of these fields in the action. Put yet another way, the equations of motion are obtained by varying the action with respect to $\phi$ and $g_{M N}$ as complex variables, as opposed to varying independently with respect to their real and imaginary parts.

With this extension, the FPs do not disappear at $\bar{\phi}_{0}=\phi_{0}$ but simply move to the complex- $\phi$ plane. The dimensions of the operators dual to the scalar field at each CFP (11) are complex conjugate of one another, $\bar{\Delta}_{0}=\Delta_{0}^{*}$. In addition, there are formally AdS solutions with metrics $g_{M N}=$ $\left\{h_{M N}, \bar{h}_{M N}\right\}$ whose complex radii (10) are also complex conjugates, $\bar{L}_{0}=L_{0}^{*}$. Since we assume that the coordinates are real, this relates the two metrics by complex conjugation $\bar{h}_{M N}=h_{M N}^{*}$. Accordingly, any quantities that can be computed holographically at the CFPs purely in terms of geometric quantities will be related by complex conjugation. These include the expectation value of Wilson loops $[39,40]$, the entanglement entropy [41], and holographic $c$

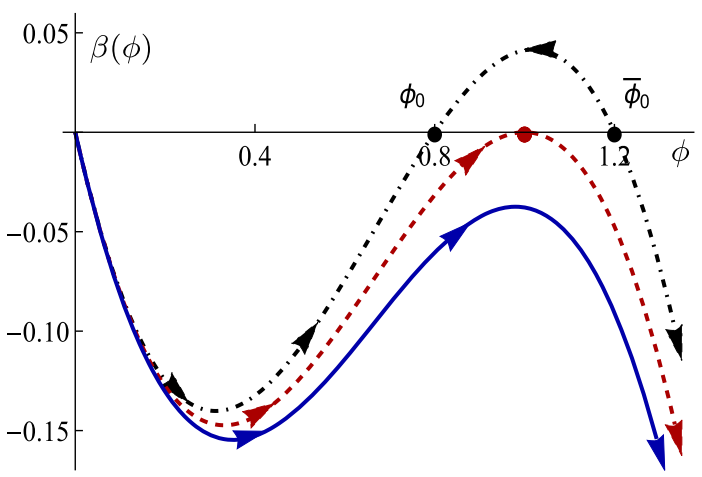

FIG. 2. $\quad \beta$ functions associated to each of the three potentials of Fig. 1, as defined in (15). The arrows indicate the direction of the $\mathrm{RG}$ flow from the UV to the IR. 
functions [42] that are related to central charges and anomaly coefficients.

This result extends to complex RG flows between the UV FP at $\phi=0$ and the CFPs. Once we continue the scalar to complex values, the first-order equations (14) split into real and imaginary parts, and the solutions describe the RG flow of the real and imaginary parts of the dual coupling. The scalar field still approaches the CFPs as given by the first equation in (16). Since both $\Delta_{c}$ and $L_{c}$ are complex the coupling oscillates. In the particular example of Fig. 3 the CFPs are IR FPs but this is not generic; i.e., CFPs can also be UV FPs. The only purely real flow is the straight horizontal line that passes exactly in between the CFPs and should exhibit walking behavior. We have collected some explicit formulas for the flows in the Supplemental Material [43].

Holographic complex conformal field theories: The holographic CFPs show many of the properties expected for a pair of CCFTs. In addition to the operator dual to $\phi$, we will show that the spectrum of dimensions of local operators at one CFP is the complex conjugate of its companion's. Let $X^{I}$ denote the real components of a field in an arbitrary Lorentz representation. Then the action expanded to quadratic order around a CFP at $\phi_{c}=$ $\left\{\phi_{0}, \bar{\phi}_{0}\right\}, h_{c}=\{h, \bar{h}\}$ will be

$$
\mathcal{L}_{c} \simeq-\sqrt{-h_{c}}\left(\frac{1}{2} X^{I} K_{c I J} X^{J}+\frac{1}{2} M_{c I J}^{2} X^{I} X^{J}\right),
$$

where we have separated a kinetic part determined by a differential operator $K_{c I J}=K_{I J}\left(h_{c}, \phi_{c}\right)=\left\{K_{I J}, \bar{K}_{I J}\right\}$ and a mass term $M_{c I J}^{2}=M_{I J}^{2}\left(h_{c}, \phi_{c}\right)=\left\{M_{I J}^{2}, \bar{M}_{I J}^{2}\right\}$. This gives the field equations

$$
K_{c I J} X_{c}^{J}+M_{c I J}^{2} X_{c}^{J}=0 .
$$

The general solution near the AdS boundary will be a superposition of exponentials of the form [44]

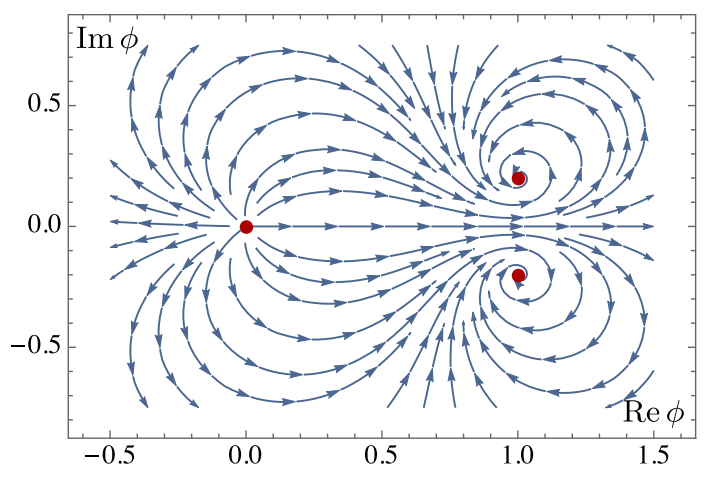

FIG. 3. Examples of complex RG flows for $d=4$ displaying the characteristic spiraling behavior around the CFPs. The flow in the UV is triggered by a source for a $\Delta_{\mathrm{UV}}=3$ operator and the CFPs are located at $\phi_{0}=\bar{\phi}_{0}^{*}=1+0.2 i$.

$$
X_{c}^{I}=\sum_{n} a_{c n}^{I} e^{-\Delta_{c n} \rho / L_{c}}+b_{c n}^{I} e^{-\left(d-\Delta_{c n}\right) \rho / L_{c}} .
$$

Introducing this in (19) one finds a homogeneous system of equations for the coefficients $a_{c n}^{I}, b_{c n}^{I}$ that has solutions when $\Delta_{c n}=\left\{\Delta_{n}, \bar{\Delta}_{n}\right\}$ take the values corresponding to the conformal dimensions of the dual operators $\mathcal{O}_{c n}=$ $\left\{\mathcal{O}_{n}, \overline{\mathcal{O}}_{n}\right\}$. We can now use holomorphicity of the action to show that

$\bar{K}=K\left(\bar{h}, \bar{\phi}_{0}\right)=K\left(h^{*}, \phi_{0}^{*}\right)=\left[K\left(h, \phi_{0}\right)\right]^{*}=(K)^{*}$,

and, similarly, that $\bar{M}^{2}=\left(M^{2}\right)^{*}$, where we have suppressed the $I J$ indices for simplicity. This implies that the spectra of operators at the two CFPs are related by complex conjugation, as anticipated:

$$
\bar{\Delta}_{n}=\Delta_{n}^{*} .
$$

Walking behavior and Miransky scaling: Our simple holographic model correctly describes the physics of walking and the associated Miransky scaling when the CFPs are close to the real axis. In particular, the $\beta$ function (15) reproduces (3). We will illustrate this scaling further by heating up the real RG flow that passes exactly in between the CFPs in Fig. 3. On the gravity side this corresponds to constructing black hole solutions that start in the UV as deformations of a $d=4$ CFT by a $\Delta_{\mathrm{UV}}=3$ operator with source $\Lambda$. We set $\phi_{0}=1+i \epsilon$ and construct solutions for several small values of $\epsilon$, following the procedure described in [45]. Some explicit technical details can be found in the Supplemental Material [43].

At very high temperature the thermodynamics is dominated by the physics of the UV CFT, hence $S_{\mathrm{UV}} \propto T^{3}$. In a region with walking the entropy would show a similar temperature scaling, so $S / S_{\mathrm{UV}}$ should be approximately constant. This plateaux can be clearly seen in the log-linear plot of Fig. 4. To quantify the size of the plateaux, we declare that the flow is in the walking region if the

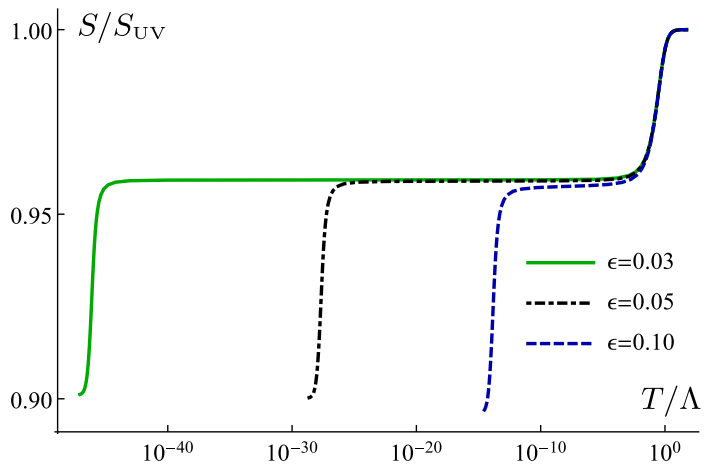

FIG. 4. Log-linear plots of the entropy density $S$ as a function of the temperature $T$, normalized to that of the UV CFT, for different values of $\phi_{0}=1+i \epsilon$. 


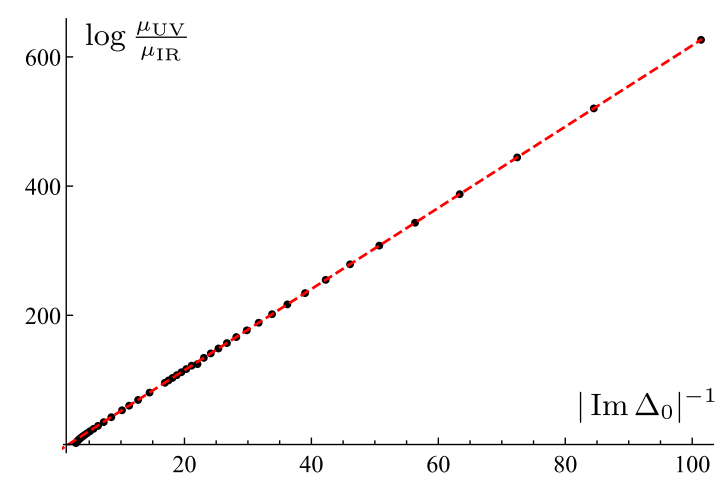

FIG. 5. Size of the walking region as a function of the imaginary part of the dimension of the operator at the CFP. The black dots are the values computed in our model while the red dashed line is a line with slope $2 \pi$ that passes through the last black dot.

following derivative is smaller than a certain control parameter $\nu$

$$
\frac{d \log \left(S / S_{\mathrm{UV}}\right)}{d \log (T / \Lambda)}<\nu
$$

For definiteness we take $\nu=\frac{1}{2} 10^{-3}$. The condition is satisfied for values of the temperature in a bounded range $T_{\mathrm{UV}}>T>T_{\mathrm{IR}}$. We identify these temperatures with the energy scales $\mu_{\mathrm{UV}}$ and $\mu_{\mathrm{IR}}$. We then vary $\epsilon$ and plot the ratio of these scales as a function of the imaginary part of the scaling dimension $\Delta_{0}$ at the CFP. The result is Fig. 5, which exhibits the expected scaling (5).

Discussion.-The simple holographic model that we have presented captures the physics of FPA. Continuing the scalar field to complex values in such a way that the action remains holomorphic, it is also possible to describe CFPs. Then a straightforward extension of the rules of the gauge/gravity duality allows us to study not only the properties of conjectured CCFTs at strong coupling but also the complex RG flows between them. When the CFP are close to the real axis the real RG flow that passes exactly between them walks and displays the associated Miransky scaling behavior.

The holomorphic gravitational action defined in this way is complex and non-Hermitian, so its meaning beyond the classical level is unclear. It would be nice to relate our proposal to those in [46,47], where it was argued that complex saddle points may give important contributions to the path integral in particular cases.

Our work shows that, contrary to some belief in the community, the RG flow leading to FPA in large- $N_{c}$ theories may be driven by a single-trace operator, the operator dual to the scalar field. Our model avoids the argument in Appendix D of [22] because the coupling constant associated to this operator is $\mathrm{O}\left(N_{c}\right)$ instead of $\mathrm{O}(1)$.

Our construction is based on a bottom-up model. It would be interesting to find top-down, string theory realizations of FPA and CFPs. While the scalar potentials obtained in consistent truncations of string and $M$ theory generically possess CFPs, an open question is whether their distance to the real axis is controlled by some parameter that can be freely varied. This issue is currently under investigation.

We are grateful to Y. Bea for collaboration in the initial stages of this project. We thank B. Fiol, I. Herbut, A. Pomarol and O. Pujolas for discussions. A. F. and D. M. are supported by MINECO Grants No. FPA2016-76005C2-1-P, No. FPA2016-76005-C2-2-P, No. MDM-20140369 and Generalitat de Catalunya Grant No. SGR2017-754. C. H. is partially supported by the MICINN Grant No. PGC2018-096894-B-100 and Principado de Asturias Grant No. GRUPIN-18-174, and the Ramon y Cajal Fellowship No. RYC-2012-10370. J. G. S. acknowledges support from the FPU program, Fellowship No. FPU15/02551.

[1] B. I. Halperin, T. C. Lubensky, and S.-k. Ma, Phys. Rev. Lett. 32, 292 (1974).

[2] B. Ihrig, N. Zerf, P. Marquard, I. F. Herbut, and M. M. Scherer, Phys. Rev. B 100, 134507 (2019).

[3] T. Senthil, A. Vishwanath, L. Balents, S. Sachdev, and M. P. A. Fisher, Science 303, 1490 (2004).

[4] A. Nahum, J. T. Chalker, P. Serna, M. Ortuño, and A. M. Somoza, Phys. Rev. X 5, 041048 (2015).

[5] C. Wang, A. Nahum, M. A. Metlitski, C. Xu, and T. Senthil, Phys. Rev. X 7, 031051 (2017).

[6] P. Serna and A. Nahum, Phys. Rev. B 99, 195110 (2019).

[7] B. Nienhuis, A. N. Berker, E. K. Riedel, and M. Schick, Phys. Rev. Lett. 43, 737 (1979).

[8] M. Nauenberg and D. J. Scalapino, Phys. Rev. Lett. 44, 837 (1980).

[9] V. Gorbenko, S. Rychkov, and B. Zan, Sci. Post. Phys. 5, 050 (2018).

[10] I. F. Herbut and L. Janssen, Phys. Rev. Lett. 113, 106401 (2014).

[11] L. Fei, S. Giombi, I. R. Klebanov, and G. Tarnopolsky, Phys. Rev. D 91, 045011 (2015).

[12] J. A. Gracey, I. F. Herbut, and D. Roscher, Phys. Rev. D 98 , 096014 (2018).

[13] T. Appelquist, D. Nash, and L. C. R. Wijewardhana, Phys. Rev. Lett. 60, 2575 (1988).

[14] K.-i. Kubota and H. Terao, Prog. Theor. Phys. 105, 809 (2001).

[15] K. Kaveh and I. F. Herbut, Phys. Rev. B 71, 184519 (2005).

[16] I. F. Herbut, Phys. Rev. D 94, 025036 (2016).

[17] H. Gies and J. Jaeckel, Eur. Phys. J. C 46, 433 (2006).

[18] E. Pomoni and L. Rastelli, J. High Energy Phys. 04 (2009) 020.

[19] D. B. Kaplan, J.-W. Lee, D. T. Son, and M. A. Stephanov, Phys. Rev. D 80, 125005 (2009).

[20] O. Antipin, S. Di Chiara, M. Mojaza, E. Mølgaard, and F. Sannino, Phys. Rev. D 86, 085009 (2012).

[21] F. F. Hansen, T. Janowski, K. Langæble, R. B. Mann, F. Sannino, T. G. Steele, and Z.-W. Wang, Phys. Rev. D 97, 065014 (2018). 
[22] V. Gorbenko, S. Rychkov, and B. Zan, J. High Energy Phys. 10 (2018) 108.

[23] V. A. Miransky, Nuovo Cimento A 90, 149 (1985).

[24] V. L. Berezinsky, Zh. Eksp. Teor. Fiz. 59, 907 (1971) [Sov. Phys. JETP 32, 493 (1971)], http://www.jetp.ac.ru/cgi-bin/ e/index/e/32/3/p493?a=list.

[25] J. M. Kosterlitz and D. J. Thouless, J. Phys. C 6, 1181 (1973).

[26] C. Sieg and M. Wilhelm, Phys. Lett. B 756, 118 (2016).

[27] D. Grabner, N. Gromov, V. Kazakov, and G. Korchemsky, Phys. Rev. Lett. 120, 111601 (2018).

[28] A. Pittelli and M. Preti, Phys. Lett. B 798, 134971 (2019).

[29] F. Benini, C. Iossa, and M. Serone, Phys. Rev. Lett. 124, 051602 (2020).

[30] K. Jensen, A. Karch, D. T. Son, and E. G. Thompson, Phys. Rev. Lett. 105, 041601 (2010).

[31] N. Iqbal, H. Liu, M. Mezei, and Q. Si, Phys. Rev. D 82, 045002 (2010).

[32] A. Pomarol, O. Pujolas, and L. Salas, J. High Energy Phys. 10 (2019) 202.

[33] M. Jarvinen and E. Kiritsis, J. High Energy Phys. 03 (2012) 002 .

[34] See however [35,36] for a holographic model whose $\beta$ function shares some properties with the lower one in our Fig. 2.
[35] J. Alanen, K. Kajantie, and K. Tuominen, Phys. Rev. D 82, 055024 (2010).

[36] J. Alanen, T. Alho, K. Kajantie, and K. Tuominen, Phys. Rev. D 84, 086007 (2011).

[37] The potential determined from (8) may have additional FPs that will play no role in our discussion.

[38] D. Anselmi, L. Girardello, M. Porrati, and A. Zaffaroni, Phys. Lett. B 481, 346 (2000).

[39] J. M. Maldacena, Phys. Rev. Lett. 80, 4859 (1998).

[40] S.-J. Rey, S. Theisen, and J.-T. Yee, Nucl. Phys. B527, 171 (1998).

[41] S. Ryu and T. Takayanagi, Phys. Rev. Lett. 96, 181602 (2006).

[42] D. Z. Freedman, S. S. Gubser, K. Pilch, and N. P. Warner, Adv. Theor. Math. Phys. 3, 363 (1999).

[43] See the Supplemental Material at http://link.aps.org/ supplemental/10.1103/PhysRevLett.124.161601 for explicit formulas for the flows and details on the construction of the black holes.

[44] For integer valued $\Delta_{c n}$ there can be additional powers of $\rho$ in the solutions, but the exponents do not change.

[45] S. S. Gubser and A. Nellore, Phys. Rev. D 78, 086007 (2008).

[46] E. Witten, arXiv:1009.6032.

[47] A. Behtash, G. V. Dunne, T. Schäfer, T. Sulejmanpasic, and M. Ünsal, Phys. Rev. Lett. 116, 011601 (2016). 